In Cres. Vol. $4 N^{\circ} 1:$ pp. 93-102, 2013

\title{
EFECTO DE ATORVASTATINA EN CÁNCER DE COLON INDUCIDO EN Rattus norvegicus var. albinus Y SU ESTUDIO HISTOPATOLÓGICO*
}

\author{
EFFECT OF ATORVASTATIN ON COLON CANCER INDUCED IN \\ Rattus norvegicus var. albinus AND ITS HISTOPATHOLOGY STUDY
}

\section{Luis Jesús Junior Guzmán Velásquez ${ }^{1}$, Suli Magali Irigoin López ${ }^{1}$, Ana María Guevara Vasquez.}

\section{RESUMEN}

El objetivo del estudio fue determinar el efecto de atorvastatina en cáncer de colon inducido en Rattus norvegicus var. Albinus. Se utilizaron 24 especímenes machos adultos divididos aleatoriamente en cuatro grupos de seis especímenes cada uno: blanco, control y problemas I y II (que recibieron $0,1143 \mathrm{mg} /$ día $/ 200 \mathrm{~g}$, equivalente a $40 \mathrm{mg} /$ día/70 $\mathrm{kg}$ y de 0,2286 $\mathrm{mg} / \mathrm{día} / 200 \mathrm{~g}$, equivalente a $80 \mathrm{mg} / \mathrm{día} / 70 \mathrm{~kg}$ de atorvastatina). Se utilizó 1,2dimetilhidrazina para inducir cáncer de colon en los grupos control y problema a la dosis de $21 \mathrm{mg} / \mathrm{kg}$ p.c/ semana, vía subcutánea durante 18 semanas. Los parámetros que se utilizaron para comprobar el efecto fueron: las observaciones histopatológicas microscópicas y macroscópicas, considerándose el número y tamaño (en milímetros) de las tumoraciones, su ubicación y el diagnóstico de tipo de lesión en el modelo experimental. Los resultados mostraron diferencia significativa entre los grupos problema y el grupo control $(p<0,01)$. La administración de atorvastatina a las dosis de $0,1143 \mathrm{mg} /$ día $/ 200 \mathrm{~g}$, equivalente a 40 $\mathrm{mg} /$ día/70 kg, y de 0,2286 mg/día, equivalente a $80 \mathrm{mg} /$ día/70 kg disminuyó el número de tumores, detuvo el crecimiento tumoral y mejoró el perfil de tipo de lesión, independientemente de la dosis administrada en Rattus norvegicus var. albinus con cáncer de colon inducido con 1,2-dimetilhidrazina.

PALABRAS ClAVE: Atorvastatina, cáncer de colon, Rattus norvegicus var. albinus, estudio histopatológico.

* Recibido: 6 de mayo del 2013; aprobado: 30 de mayo del 2013.

1 Químico Farmacéutico. Universidad Nacional de Trujillo-Perú.

2 Químico Farmacéutico, Doctora en Ciencias Biomédicas, Docente de la Universidad Nacional de Trujillo-Perú. 


\begin{abstract}
The aim of the study was to determine the effect of atorvastatin in colon cancer induced in albino rats. For this purpose, 24 adult males of Rattus norvegicus var. albinus were randomly divided into four groups of six specimens each: white, control and problems I and II (that received $0,1143 \mathrm{mg} /$ day/200 g equivalent $40 \mathrm{mg} /$ day $/ 70 \mathrm{~kg}$ and $80 \mathrm{mg} / \mathrm{day} / 70 \mathrm{~kg}$ equivalent $0,2286 \mathrm{mg} / \mathrm{day} / 200 \mathrm{~g}$ to atorvastatin). 1,2-dimethylhydrazine was used to induce colon cancer in control and problem groups to doses of $21 \mathrm{mg} / \mathrm{kg} \mathrm{bw} /$ week subcutaneously for 18 weeks. The parameters used to test the effect were: microscopic and macroscopic histopathological findings considering the number and size (in millimeters) of tumors, location and diagnosis of type of injury in an experimental model. The results showed a significant difference between problem and control groups $(\mathrm{p}<0,01)$. In conclusion the doses of atorvastatin decreased the number of tumors, tumor growth arrest and improved the profile of type of injury in a doses-independent way in Rattus norvegicus var. albinus with colon cancer induced by 1,2-dimethylhydrazine.
\end{abstract}

KEY WORDS: Atorvastatin, colon cancer, Rattus norvegicus var. albinus, histopathological study.

\title{
I. INTRODUCCIÓN
}

El cáncer de colon es el cuarto diagnóstico más frecuente y la segunda principal causa de muerte de hispanos; representa la sexta causa de mayor frecuencia de cáncer en el norte de Perú. Asimismo, muestra una marcada incidencia y mortalidad para ambos sexos, siendo un problema de salud humana. ${ }^{1,2}$

Estudios actuales evalúan la relación entre el uso de estatinas y riesgo de cáncer. Los primeros estudios realizados en modelos animales expresaron su preocupación de que las estatinas podrían tener propiedades cancerígenas. Una revisión de los hallazgos de carcinogenicidad en roedores por fármacos hipolipemiantes reveló que todas las estatinas disponibles en 1994, iniciaban o promovían el cáncer a concentraciones equivalentes a las que comúnmente son prescritos en los seres humanos. ${ }^{3,45}$

Contrariamente a las inquietudes sobre la carcinogenicidad de las estatinas, la creciente evidencia sugiere que pueden tener un potencial efecto quimiopreventivo contra el cáncer. Por tanto, la relevancia clínica y mecanicista de los estudios en animales y seres humanos de las estatinas y el cáncer no está esclarecido. ${ }^{6}$ Entonces, ante la falta de evidencia consistente en el riesgo de cáncer sobre órgano específico y los diversos fármacos presentes en el grupo terapéutico de los inhibidores de la enzima hidroximetilglutaril CoA reductasa (HMGCoA reductasa), se ha determinado el efecto de atorvastatina mediante un estudio histopatológico sobre un modelo experimental de cáncer de colon, y así con- 
tribuir con nuevos aportes dentro de este campo entre las asociaciones de estatinas y el riesgo de cáncer.

\section{PROBLEMA}

¿Qué efecto tiene la administración de atorvastatina en cáncer de colon inducido en Rattus norvegicus var. albinus?

\section{HIPÓTESIS}

Atorvastatina disminuye el número de tumores, detiene el crecimiento tumoral y mejora el perfil de tipo de lesión en cáncer de colon en Rattus norvegicus var. albinus.

\section{OBJETIVO}

Determinar el efecto de atorvastatina en cáncer de colon inducido en Rattus norvegicus var. albinus mediante su estudio histopatológico.

\section{MATERIAL Y MÉTODO}

\subsection{MATERIAL}

\section{a) Material farmacológico}

Se utilizaron 30 tabletas de atorvastatina de $10 \mathrm{mg}$ (Lab. AC FARMA'), las cuales se pulverizaron mediante molienda para las dosis. Esta actividad fue realizada semanalmente para evitar pérdida de estabilidad del principio activo.

\section{b) Material biológico}

Se utilizaron 24 especímenes de Rattus norvegicus var. albinus, machos adultos, desparasitados y con peso promedio de $200 \mathrm{~g}$, que fueron adquiridos en el Instituto Nacional de Salud, Chorrillos, Lima, y alojados en el Bioterio de la Facultad de Farmacia y Bioquímica de la Universidad Nacional de Trujillo bajo condiciones de ciclo luz/ oscuridad de 12/12 horas, temperatura ambiental entre 22 y $24{ }^{\circ} \mathrm{C}$ y alimentados con dieta estándar y agua ad libitum. Todos los procedimientos fueron realizados acordes con los protocolos aprobados por la Institución y la Guía para el Cuidado y Uso de Animales de Laboratorio (NRC, USA 1996).

\subsection{MÉTODO}

a) Inducción de cáncer de colon con 1,2-dimetilhidrazina y administración de atorvastatina en Rattus norvegicus var. albinus. Se trabajó 
con cuatro grupos: un grupo blanco, un grupo control y dos grupos problema, constituidos por 6 especímenes de Rattus norvegicus var. albinus cada uno, distribuidos en forma aleatoria.

Grupo I (Blanco). No recibió tratamiento alguno; sólo la solución salina fisiológica (SSF) vía subcutánea en la zona lumbar izquierda, una vez por semana, durante 18 semanas y, luego, a partir de la semana 19, se administró SSF por vía oral, mediante sonda de alimentación hasta la semana 32 .

\section{Inducción de cáncer de colon con 1,2-dimetilhidrazina ${ }^{4}$}

Las ratas albinas fueron inyectadas semanalmente con 1,2dimetilhidrazina (DMH) subcutáneamente en la zona lumbar izquierda a la dosis de $21 \mathrm{mg} / \mathrm{kg}$ de peso corporal. La DMH fue disuelta en solución salina fisiológica $(10 \mathrm{mg} / \mathrm{mL})$.

Grupo II (Control). Se les inyectó semanalmente una dosis de DMH, según técnica descrita, desde el principio del estudio, durante 18 semanas; y luego, a partir de la semana 19, se administró SSF por vía oral, mediante sonda de alimentación hasta la semana 32.

Grupo III (Problema I). Se les inyectó DMH, según técnica descrita, durante 18 semanas, por vía subcutánea, desde el principio del estudio; y, luego, a partir de la semana 19, se administró atorvastatina por vía oral, mediante sonda de alimentación a la dosis de $0,1143 \mathrm{mg} /$ día $/ 200 \mathrm{~g}$ equivalente a $40 \mathrm{mg} /$ día/70 $\mathrm{kg}$ hasta la semana 32 .

Grupo IV (Problema II). Se les inyectó DMH, según técnica descrita, durante 18 semanas, por vía subcutánea, desde el principio del estudio; y luego, a partir de la semana 19 , se administró atorvastatina por vía oral, mediante sonda de alimentación a la dosis de 0,2286 mg/día/200 g equivalente a $80 \mathrm{mg} /$ día $/ 70 \mathrm{~kg}$ hasta la semana 32 .

b) Demostración del efecto de atorvastatina en Rattus norvegicus var. Albinus. Se realizó un estudio histopatológico macroscópico y microscópico, considerándose el número y tamaño (en milímetros) de las tumoraciones, su ubicación y en base al diagnóstico de tipo de lesión, considerándose: adenoma, adenocarcinoma In situ, adenocarcinoma glandular y adenocarcinoma escamoso, presentes en el modelo experimental. 


\subsection{ANÁLISIS ESTADÍSTICO}

Los datos de número y tamaño de tumores fueron expresados como promedio y desviación estándar y analizados estadísticamente tomando como parámetro central la media aritmética; se calculó la desviación estándar y el estudio comparativo se realizó mediante la distribución t de Student. ${ }^{7}$ Para inferir estadísticamente en base a los resultados se consideró un nivel de significancia de 0,05 .

\section{RESULTADOS}

\section{Tabla 01}

OBSERVACIONES MACROSCÓPICAS Y MICROSCÓPICAS EN

Rattus norvegicus var. albinus

\begin{tabular}{lccccc}
\hline Grupos & R & N & TP(mm) & Ubicación & Tipo de lesión \\
\hline BLANCO & 0 & 0 & 0 & s.d. & s.d. \\
CONTROL & 6 & $9,16 \pm 1,5$ & $8,5 \pm 1,14$ & $\begin{array}{c}\text { Colon medio } \\
y \text { distal }\end{array}$ & $\begin{array}{c}\text { Adenocarcinoma } \\
\text { Glandular }\end{array}$ \\
PROBLEMA I & 6 & $1,67 \pm 0,82^{*}$ & $5,67 \pm 1,03^{* *}$ & $\begin{array}{c}\text { Colon distal } \\
\text { Adenoma }\end{array}$ & Adenoma \\
\hline PROBLEMA II & 5 & $1,33 \pm 0,81^{*}$ & $5,17 \pm 2,75^{* *}$ & Colon distal & Ad
\end{tabular}

(R): número de ratas con tumoraciones; (N): número de tumoraciones promedio; (TP): tamaño promedio de tumoraciones; $\mathrm{n}=6$ especímenes; ${ }^{*} \mathrm{p}<0,01,{ }^{* *} \mathrm{p}<0,05$; Problemal (Atorvastatina: 0,1143 mg/día/200 g); II (Atorvastatina: 0,2286 mg/día/200 g). s.d.: sin datos.

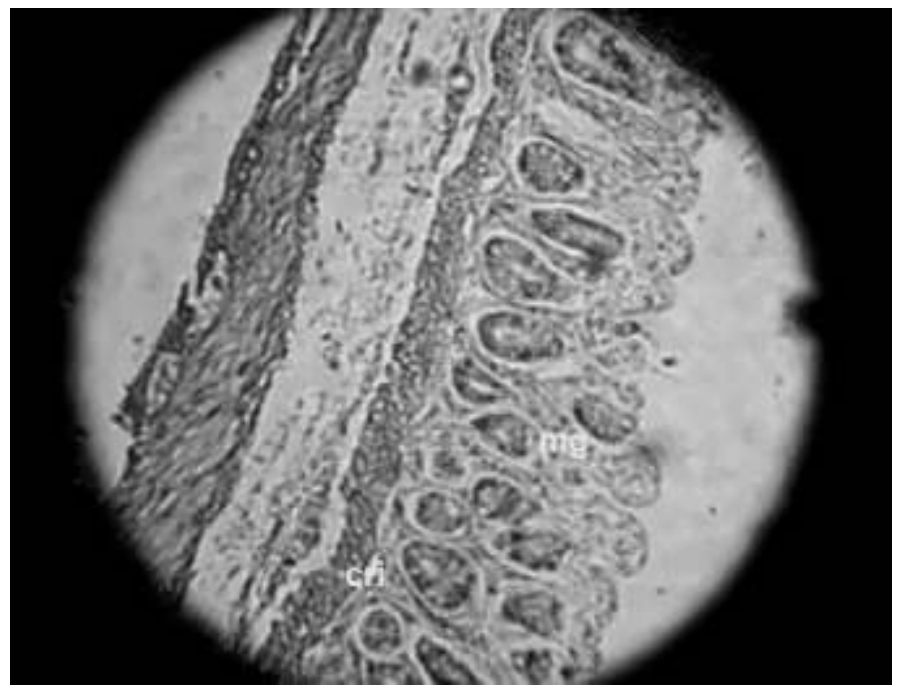

Figura 1. Grupo blanco. Corte histológico de colon distal de Rattus norvegicus var. albinus. mg): glándulas mucosas, cri): criptas de Lieberkuhn.

Estructuras normales de corte histológico de colon. HE. 10x. 


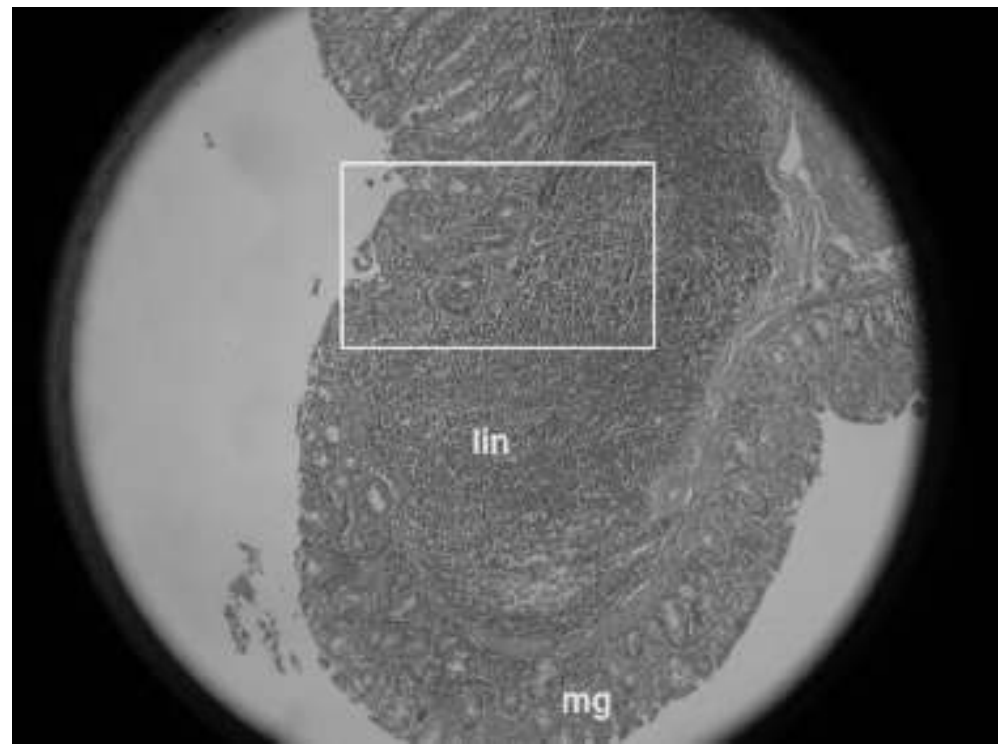

Figura 2. Grupo control. Corte histológico de colon distal de Rattus norvegicus var. albinus. Lin): agregados linfoides, mg): glándulas mucosas.

Discontinuidad de la mucosa epitelial (recuadro). HE. 40x.

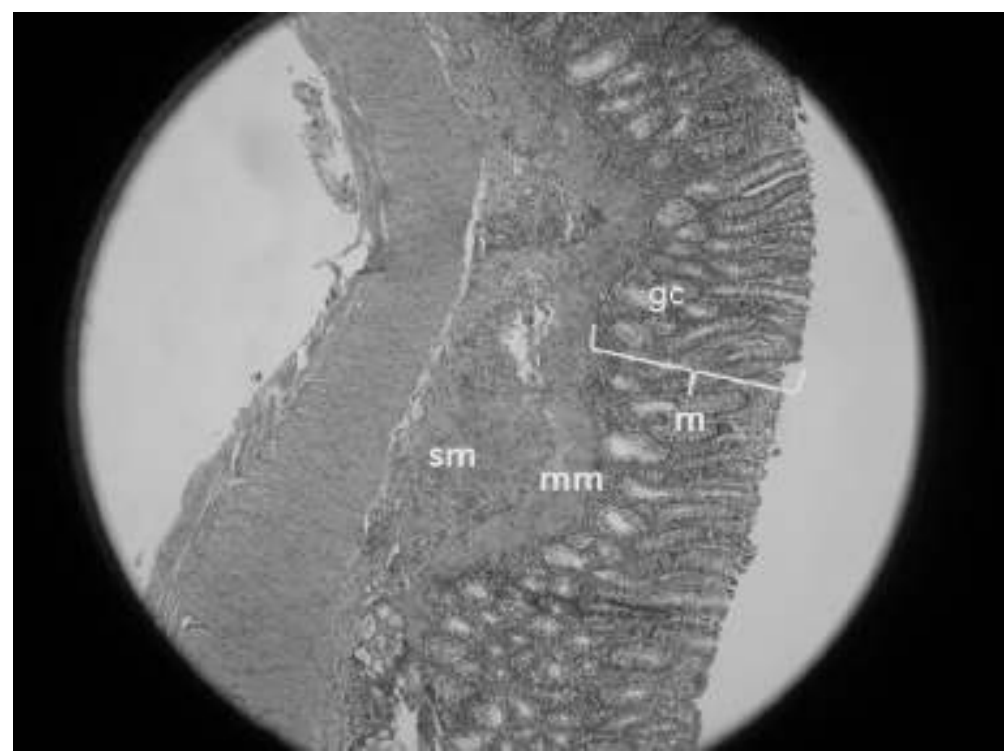

Figura 3. Grupo problema I. Corte Histológico de Colon Distal de Rattus norvegicus var. albinus. Detalle de mucosa normal (m), criptas mostrando glándulas colónicas (gc) activas. muscular de la mucosa (mm) y la submucosa (sm). H\&E 100X. 


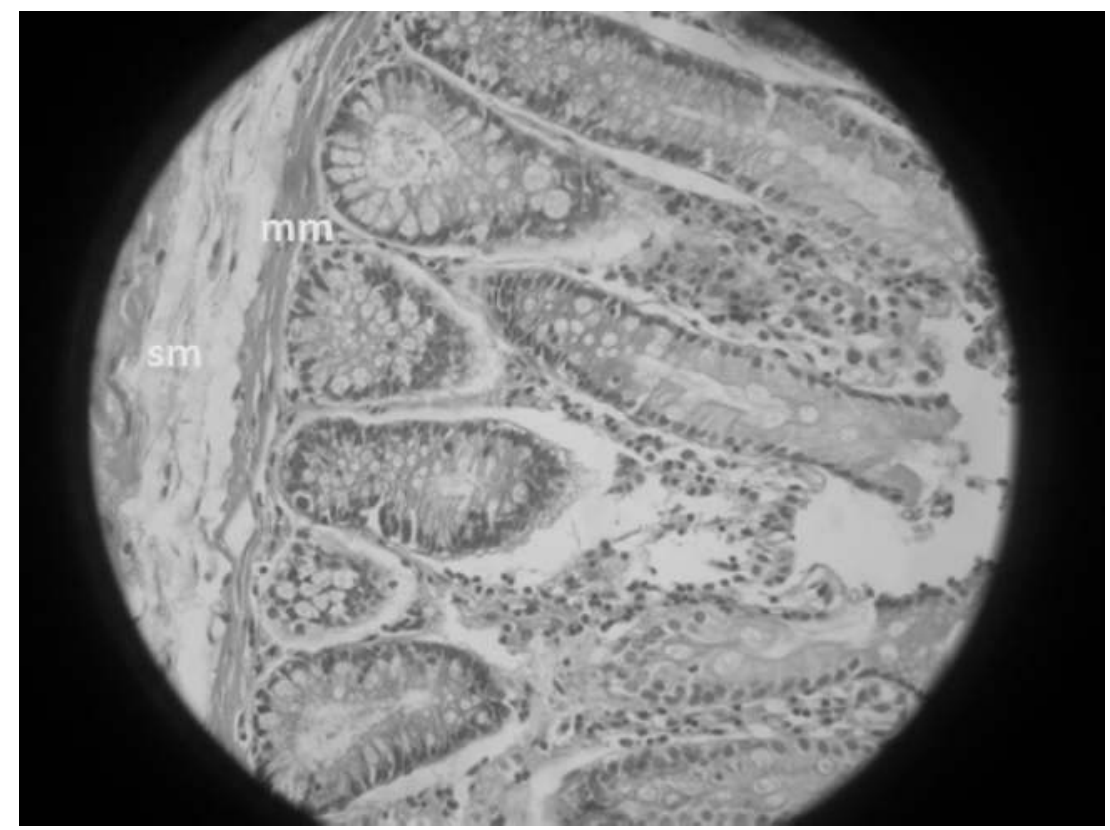

Figura 4. Grupo problema II. Corte Histológico de Colon Distal de Rattus norvegicus var. albinus. Detalle de mucosa glandular con epitelio de revestimiento y glándulas colónicas activas recuperadas, muscular de la mucosa (mm) y submucosa (sm) con tejido conectivo, normales. H\&E 400X.

\section{DISCUSIÓN}

El grupo terapéutico de los inhibidores de la HMG-CoA reductasa es un potente inhibidor de la síntesis de colesterol, a nivel clínico, su utilización es de gran beneficio en la prevención de primaria y secundaria de la enfermedad cardiaca coronaria. Sin embargo, los inhibidores poseen múltiples actividades independientes de la inhibición de metabolismo de lípidos, tales como: mejorar la disfunción endotelial, inhibir la inflamación vascular, inhibir la trombosis y disminuir el estrés oxidativo. Estas acciones se conocen como "efectos pleiotrópicos" de las estatinas; por lo tanto, los efectos beneficiosos de estos fármacos no sólo serían observables en pacientes hiperlipidémicos. ${ }^{8,9,10}$

Tras determinar el número y tamaño de las tumoraciones presentes en el modelo experimental se observa que estos cambios en la morfología celular colónica, producto de la inducción experimental con DMH, se fundamentan en la capacidad carcinogénica, órgano selectivo del agente alquilante metildiazonio derivado del metabolismo de DMH, lo que conduce a alteraciones en las 
propiedades de macromoléculas críticas como el ADN, ARN y otras proteínas en el epitelio intestinal, ocasionando alteraciones en el fenotipo celular, tales como pérdida de diferenciación, invasividad o conducta proliferativa anormal. Esta inestabilidad genómica, principalmente inestabilidad cromosómica, causa cambios en el número de copias de cromosomas y sus estructuras, lo suficiente para generar una pérdida física de las copias dominantes de los genes supresores de tumores, tales como como $A P C, P 53$ y SMAD4. Asimismo, las mutaciones oncogénicas del RAS (K-RAS) que activan la vía de señalización de la proteína kinasa, activada por mitógenos (MAPK), desempeñan un papel clave en la promoción del cáncer de colon. ${ }^{4,11,12}$

Se conoce que las modificaciones postraduccionales de las proteínas de la superfamilia Ras son metabolizadas por preniltrasnferasas citosólicas que añaden un grupo polisoprenoide (farnesil y geranilgeranil) a secuencias especificas dentro de su estructura, indispensables para el anclaje en la membrana plasmática. Tras el tratamiento de los grupos problemas con atorvastatina se previene la conversión de la HMG-CoA a mevalonato y, por lo tanto, reducen los niveles de mevalonato y sus productos derivados, necesarios para la prenilación del KRas. ${ }^{13}$ Por tanto, la interrupción de estos procesos en las células neoplásicas puede resultar en un control de la iniciación del tumor, el crecimiento y su invasividad. Como se observa en la Tabla 1, existe una similitud de la afección carcinogénica con respecto al número de tumoraciones promedio de los grupos problema I $(1,67 \pm 0,82 \mathrm{~mm})$ y II $(1,33 \pm 0,81 \mathrm{~mm})$; pero, con respecto al número de tumoraciones del grupo control $(9,16 \pm 1,5 \mathrm{~mm})$, el número de tumoraciones de ambos grupos-problema es significativamente menor $(\mathrm{p}<0,01)$. Asimismo, se observa que el tamaño promedio de tumoraciones tiende a disminuir para los grupos problema, resultando también significativa $(\mathrm{p}<0,05)$.

Tras la evaluación histopatológica microscópica se observaron los cambios celulares de las estructuras glandulares de la capa interna del colon, en condiciones normales, las glándulas del colon, denominadas glándulas de Lieberkuhn, tienden a ser de apariencia simple y tubular, y el epitelio que las forma es cilíndrico simple (Figura1). Sin embargo, la evolución carcinogénica en el modelo experimental para los tratamientos con atorvastatina muestra lesiones premalignas, las cuales se caracterizan por displasia epitelial, anomalías núcleo-citoplasmáticas y cambios de la arquitectura de las glándulas con una ligera reacción en la muscular de la mucosa y una leve reacción inflamatoria en la submucosa (Figura 3). Tras el aumento de dosis de atorvastatina administra- 
da, se observa una mucosa glandular con revestimiento epitelial y glándulas colónicas activas recuperadas, la muscular de la capa mucosa, capa submucosa y tejido conectivo normales (Figura 4). Estas características contrasta con la neoplasia maligna del epitelio glandular, en la cual se aprecia la discontinuación de la muscular de la capa mucosa que abarca la capa submucosa asociada a agregados linfoides presentes en la misma (Figura 2). Existe evidencia que atorvastatina, tras actuar como limitante en la síntesis de colesterol, y debido a sus características pleiotrópicas, posee un potencial beneficio dentro de los procesos carcinogénicos, tal como se observa en el modelo experimental. ${ }^{12,13,14}$

\section{CONCLUSIONES}

Atorvastatina disminuye el número de tumores $(\mathrm{p}<0,01)$, detiene el crecimiento tumoral $(\mathrm{p}<0,05)$ y mejora el perfil de tipo de lesión (adenoma) a dosis de $0,1143 \mathrm{mg} /$ día, equivalente a $40 \mathrm{mg} /$ día/ $70 \mathrm{~kg}$ y de $0,2286 \mathrm{mg} /$ día equivalente a $80 \mathrm{mg} / \mathrm{día} / 70 \mathrm{~kg}$. No existe diferencia significativa entre grupos problema en Rattus norvegicus var. albinus con cáncer de colon inducido con 1,2dimetilhidrazina.

\section{CONFLICTO DE INTERESES}

Los autores declaran que no existe ningún conflicto de interés relacionado con el artículo.

\section{REFERENCIAS BIBLIOGRÁFICAS}

1 Siegel R, Naishadham D, Jemal A. 2012. Cáncer statistics for hispanics/latinos. CA: A cancer journal for clinicians. Vol. 62, $\mathrm{N}^{\circ}$ 5: 283-298.

2 DíAZ J, HERnÁNDEZ P, BuRga A. 2012. Registro hospitalario de cáncer. Indicadores hospitalarios Año 2012. Instituto regional de enfermedades neoplásicas - Norte. Servicio de epidemiologia y estadística.

3 Benson A, BeKait T, Chan E, et al. 2013. Colon Cancer. National comprehensive cancer network. NCCN Clinical practice guidelines in oncology. Version 3.

4 PiÑols, C. 1999. Estudios de los cambios de la mucosa colónica de rata y cinética celular durante la carcinogénesis experimental inducida con 1,2-dimetilhidrazina. Universitat de Lleida. Salamanca. España. Pp.16; 23-25.

5 Newman T, Hulley S. 1996. Carcinogenicity of lipid lowering drug. The journal of Americam Medical Association. 1996. Vol. 275, $\mathrm{N}^{\circ}$ 1: 55-60.

6 BROWNING D, MARTIN R. 2007. Statin and risk of cancer: a systematic review and meta-analysis. International Journal of Cancer. Vol. 120, $\mathrm{N}^{\circ}$ 4: 833-843.

7 WAYNe W, DANIEL. 2004. Bioestadística. Base para el análisis da las ciencias de la salud. $4^{\mathrm{a}}$ ed. Limusa Wiley. México. 41-42. 
8 AdAm J, AKehURST R, ANGRIS S, et al. 2008. National Institute for Health and Clinical Excellence (NICE). Statin for the prevention of cardiovascular events. London.

9 Mennickent S, Bravo M, Calvo C, Avello M. 2008. Efectos pleiotrópicos de las estatinas. Revista Médica de Chile. Vol. 136, $\mathrm{N}^{\circ}$ 6: 775-782.

10 Liao J, LAUfS U. 2005. Pleoitropic effects of statins. Annual Review Pharmacology and Toxicology. Vol.45: 89-118.

11 MARKowitz S, Bertagnolli M. 2009. Molecular basis of colorectal cancer. The New England Journal of Medicine. Vol. 336: 2449-2460.

12 LOPEZ C. 2006. Regulación de K-Ras por $\mathrm{Ca}^{2+} / \mathrm{CaM}$. Universitat de Barcelona. Tesis para optar grado de Doctor en Farmacia. España.

13 FinN O. 2008. Molecular Origens of cáncer: Cancer inmunology. The New England Journal of Medicine. Vol. 358: 2704-2715.

14 Colina F, IBARrola C. Protocolo e información sistematizada para los estudios histopatológicos relacionados con el carcinoma colorrectal. Revista Española de Patologia. 2004. Vol. 37, $\mathrm{N}^{\circ} 1$ : 73-90.

Correspondencia: luisjesusg29@gmail.com 